pacientes infectados por VIH en un hospital público peruano. Rev Peru Med Exp Salud Publica. 2017;34(2):239-44. doi: 10.17843/rpmesp.2017.342.2587.

2. Bewick V, Cheek L, Ball J. Statistics review 14: Logistic regression. Crit Care. 2005;9(1):112-8. doi: 10.1186/cc3045.

3. Warner P. Poisson regression. J Fam Plann Reprod Health Care. 2015;41(3):223-4. doi: 10.1136/jfprhc-2015-101262.

4. Coutinho LM, Scazufca M, Menezes PR. Methods for estimating prevalence ratios in cross-sectional studies. Rev Saude Publica. 2008;42(6):992-8.

5. McNutt LA, Wu C, Xue X, Hafner JP. Estimating the Relative Risk in Cohort Studies and Clinical Trials of Common Outcomes. Am J Epidemiol. 2003;157(10):940-3.

Correspondencia: Reyna Farfan García

Dirección: Av. Alameda San Marcos $s / n$, Distrito de Chorrillos, Perú Teléfono: (+51) 01-313-3333

Correo electrónico: reyna.farfan16@gmail.com

\section{¿SON MENOS DAÑINAS LAS GASEOSAS SIN AZÚCAR? UN ANÁLISIS DE LA INFORMACIÓN NUTRICIONAL EN DOS CIUDADES PERUANAS}

\section{ARE SUGAR-FREE SOFT DRINKS LESS HARMFUL? AN ANALYSIS OF THE NUTRITIONAL INFORMATION IN TWO PERUVIAN CITIES}

\section{Diego Azañedo ${ }^{1, a}$, Lorena Saavedra-Garciaª, ${ }^{2,3, b}$, Juan Carlos Bazo-Alvarez ${ }^{1,4, c}$}

Sr. Editor. La mortalidad mundial por enfermedades cardiovasculares (ECV) se estima en aproximadamente 18 millones de muertes anuales, llegando a representar casi la mitad del total de muertes atribuidas a las enfermedades no transmisibles (ENT). Por ello, los organismos internacionales han tomado diversas medidas para su reducción, como el Plan de Acción Global para la Prevención y Control de Enfermedades no Transmisibles, impulsado por la

\footnotetext{
Centro de Estudios de Población, Universidad Católica Los Ángeles de Chimbote (ULADECH-Católica). Chimbote, Perú.

2 CRONICAS Center of Excellence in Chronic Diseases, Universidad Peruana Cayetano Heredia. Lima, Peru.

Escuela de Nutrición y Dietética, Facultad de Ciencias de la Salud, Universidad Científica del Sur. Lima, Peru.

4 Methodology Research Group, Department of Primary Care and Population Health, University College London (UCL). London, UK.

a Cirujano dentista; ${ }^{b}$ nutricionista, máster en alimentación, nutrición y metabolismo; ${ }^{\mathrm{c}}$ licenciado en Psicología, maestro en Ciencias en Investigación Epidemiológica.

Recibido: 26/10/2017 Aprobado: 07/02/2018 En línea:05/04/2018
}

Citar como: Azañedo D, Saavedra-Garcia L, Bazo-Alvarez JC. ¿Son menos dañinas las gaseosas sin azúcar? un análisis de la información nutricional en dos ciudades peruanas. Rev Peru Med Exp Salud Publica. 2018;35(1):164-6. doi: 10.17843/rpmesp.2018.351.3555.
Organización Mundial de la Salud (OMS), que tiene como uno de sus objetivos reducir en $25 \%$ la mortalidad prematura por ECV, cáncer, diabetes y enfermedad pulmonar obstructiva crónica para el año $2025^{(1)}$.

El consumo excesivo de azúcares presentes en alimentos ultraprocesados como las gaseosas incrementan el sobrepeso y la obesidad, y con ello el riesgo de desarrollar ECV ${ }^{(2)}$. Sin embargo, la aparición en el mercado de las gaseosas "libres de azúcar" (versiones light o zero), como una alternativa "menos dañina", ha despertado el interés y preferencia de los consumidores ${ }^{(3)}$. Estas últimas reemplazan el azúcar por edulcorantes no nutritivos como estevia, aspartame, acesulfame de potasio o sacarina; disminuyendo la carga energética del producto. No obstante, no se ha prestado atención a la variación en otro de sus nutrientes críticos como el sodio.

Es conocido que una alimentación con elevados niveles de sodio en los alimentos y bebidas, es uno de los principales factores de riesgo para que se produzcan ECV ${ }^{(4)}$. Debido a ello, en el año 2010, la Asociación Americana del Corazón recomendó un máximo consumo diario de sodio de $1,5 \mathrm{~g}$ para adultos con hipertensión y otros factores de riesgo, y de 2,3 g para adultos sanos ${ }^{(4)}$. A pesar de dicha recomendación, la media de consumo de sodio en países de América Latina aún supera los $4 \mathrm{~g}$ (equivalente a $10 \mathrm{~g}$ de sal) ${ }^{(5)}$.

El consumo de bebidas gaseosas es elevado en el Perú. Según el Instituto Nacional de Estadística e Informática (https://goo.gl/pFNw93) en el 2010, el consumo promedio per cápita anual de gaseosas en el Perú fue de 30,6 litros en la costa, 24,8 litros en la sierra y 22,9 litros en la selva. Asimismo, considerando el quintil de ingresos, existen grandes variaciones $(47,1 \mathrm{~L}$ en el quintil 5 versus $8,5 \mathrm{~L}$ en el quintil 1) que indican una concentración del consumo en la población de mayores recursos.

Por todo lo anterior, se realizó una revisión de la información nutricional publicada en las bebidas gaseosas que se ofrecen en establecimientos comerciales de dos ciudades peruanas, Chimbote y Lima. Se recolectaron y revisaron las etiquetas de las botellas de $500 \mathrm{ml}$ de cada una de las siguientes marcas comerciales de gaseosa: Pepsi, Coca Cola, Sprite, Fanta e Inka Kola; tanto en su versión regular, como en su versión "libre de azúcar". Se estandarizó las unidades de medida de energía (Kcal), carbohidratos totales (g), azúcar (g) y sodio (mg), por cada $100 \mathrm{ml}$ de la bebida; elaborando luego una tabla comparativa (Tabla 1).

Los resultados muestran que, a pesar de la ausencia de azúcar en las bebidas "light o Zero", una de ellas (Coca Cola Zero) casi duplica y otra (Fanta Zero) triplica el contenido de sodio de su par azucarada. Por ejemplo, si una persona tomara una Fanta de $500 \mathrm{ml}$ en el almuerzo y otra en la cena estaría consumiendo $79 \mathrm{mg}$ de sodio, si la misma 
Tabla 1. Información nutricional por cada 100 ml de las bebidas gaseosas, en su versión regular y “libre de azúcar" en Perú

\begin{tabular}{lcccc}
\hline Bebida gaseosa & Energía (kcal) & Carbohidratos totales (g) & Azúcar (g) & Sodio (mg) \\
\hline Pepsi & 44,0 & 11,0 & 11,0 & 5,0 \\
Pepsi light & 0,0 & 0,0 & 0,0 & 4,6 \\
Coca Cola & 44,0 & 10,8 & 10,8 & 5,8 \\
Coca Cola Zero & 0,0 & 0,0 & 0,0 & 10,4 \\
Sprite & 40,0 & 10,0 & 10,0 & 7,9 \\
Sprite Zero & 0,0 & 0,0 & 0,0 & 6,7 \\
Fanta & 52,0 & 12,9 & 12,9 & 7,9 \\
Fanta Zero & 0,0 & 0,0 & 0,0 & 25,8 \\
Inca Kola & 42,0 & 10,5 & 10,5 & 9,0 \\
Inca Kola Zero & 0,0 & 0,0 & 0,0 & 9,0 \\
\hline
\end{tabular}

persona consumiera Fanta Zero de $500 \mathrm{ml}$, el consumo de sodio aumentaría a $258 \mathrm{mg}$ ( $226 \%$ más sodio), si este nivel de consumo se realizara todos los días durante un año, equivaldría a 45,6 cucharaditas de sal. Por otro lado, si otra persona decidiera consumir Coca Cola de $500 \mathrm{ml}$ en la misma proporción, estaría consumiendo 58 mg de sodio; pero, si consumiera la misma cantidad de Coca Cola Zero, equivaldría a $104 \mathrm{mg}$ de sodio (79 \% más sodio), y si este nivel de consumo se realizara todos los días durante un año equivaldría a 18,3 cucharaditas de sal. En este sentido, la versión "Zero" de ambas marcas de gaseosa deja de ser menos dañina.

El consumidor debe comprender que la reducción de la cantidad de azúcar de las gaseosas no garantiza que el sodio se mantenga en su misma proporción. De hecho, el etiquetado actual de estos productos no facilita este mensaje al consumidor. Por ejemplo, cada marca indica la composición nutricional de distinta manera, algunas por cada $100 \mathrm{ml}$, otras en contenido total por envase y otras en porciones, las cuales pueden variar $(200 \mathrm{ml}, 237 \mathrm{ml}$ o $240 \mathrm{ml}$ ) a pesar que se trata de bebidas del mismo tipo. Situación contraria ocurre en países desarrollados, como Inglaterra, donde se obliga a las empresas a presentar información nutricional en un formato estándar (100 g o $100 \mathrm{ml}$ ); haciendo que la información sea más fácil de comprender y comparar ${ }^{(6)}$. Por ello, es importante que en Perú la información también se comunique de forma sencilla y clara. Creemos que recibir esta información en forma estandarizada, es un derecho de los consumidores.

En ese sentido, se recomienda a los usuarios evaluar detalladamente la información nutricional de los alimentos o bebidas que consumen. Asimismo, se hace un llamado a la Asociación Peruana de Consumidores y Usuarios, al Instituto Nacional de Defensa de la Competencia y de la Protección de la Propiedad Intelectual y al Ministerio de la Producción para que aseguren la implementación de un formato estándar de etiquetado que brinde información de manera más amigable a los consumidores, permitiéndoles tomar decisiones informadas al momento de seleccionar los alimentos que consumirán. Por otro lado, los ministerios de salud y educación deben focalizar esfuerzos en educar al consumidor desde la niñez en la lectura e interpretación del etiquetado nutricional de los productos, así como en promover el consumo de alimentos saludables. Estamos seguros de que la adopción de estas medidas contribuirá a la mejora del perfil nutricional de la población y, como consecuencia, a la reducción de enfermedades crónicas no transmisibles y sus complicaciones.

Contribuciones de autoría: DA Y JCBA han participado en la concepción del manuscrito, recolección e interpretación de los datos; DA, JCBA Y LSG han participado de la redacción, revisión crítica y aprobación de la versión final del manuscrito.

Fuente de financiamiento: el presente estudio ha sido financiado por la Universidad Católica los Ángeles de Chimbote

Conflictos de interés: los autores declaran no tener conflictos de interés con respecto a este artículo

\section{REFERENCIAS BIBLIOGRÁFICAS}

1. Sacco RL, Roth GA, Reddy KS, Arnett DK, Bonita R, Gaziano TA, et al. The Heart of 25 by 25: Achieving the Goal of Reducing Global and Regional Premature Deaths From Cardiovascular Diseases and Stroke. A Modeling Study From the American Heart Association and World Heart Federation. Glob Heart. 2016;11(2):251-64. doi: 10.1016/j.gheart.2016.04.002.

2. Rippe JM, Angelopoulos TJ. Sugars, obesity, and cardiovascular disease: results from recent randomized control trials. Eur J Nutr. 2016;55(Suppl 2):45-53. doi: 10.1007/s00394-016-1257-2.

3. Piernas C, Ng SW, Popkin B. Trends in purchases and intake of foods and beverages containing caloric and low-calorie sweeteners over the last decade in the U.S. Pediatr Obes. 2013;8(4):294-306. doi: 10.1111/j.2047-6310.2013.00153.x.

4. Lloyd-Jones DM, Hong Y, Labarthe D, Mozaffarian D, Appel LJ, Van Horn L, et al. Defining and setting national goals for cardiovascular health promotion and disease reduction: the American Heart Association's strategic Impact Goal through 2020 and beyond. Circulation. 2010;121(4):586-613. doi: 10.1161/ CIRCULATIONAHA.109.192703.

5. Panamerican health organization. Countries of the Americas are Taking Action to Reduce Salt Consumption and Save Lives 
[Internet]. Washington, D.C., USA: PAHO; 2011 [actualizado el 11 de noviembre; citado el 3 de mayo de 2017]. Disponible en: http://www.paho.org/hq/index.php?option=com_content\&vie $\mathrm{w}=$ article\&id $=6152 \% 3 \mathrm{~A} 2011$-countries-americas-taking-actionreduce-salt-consumption-save-lives\&Itemid=1926\&lang=en.

6. Population health Division. Technical guidance on nutrition labelling. In: Department of health. London, UK: UK goverment; 2017.p. 22.

Correspondencia: Diego Azañedo

Dirección: Jr. Tumbes 247, Casco urbano, Chimbote, Ancash, 02804, Perú. Teléfono: (43) 947121972

Correo electrónico:dazanedov@uladech.edu.pe

\section{PRODUCCIÓN CIENTÍFICA EN BASE A CINCO ENCUESTAS NACIONALES DE PERÚ}

\section{SCIENTIFIC PRODUCTION BASED ON FIVE NATIONAL SURVEYS IN PERU}

\author{
Juan C. Ruiz-Maza ${ }^{1, a}$, Armando M. Pezo-Pezo, a, \\ Renato A. Soto-Azpilcueta ${ }^{1, a}$
}

Sr. Editor. El Instituto Nacional de Estadística e Informática (INEI) ejecuta encuestas nacionales que recogen información sobre temas específicos como la salud reproductiva materno infantil y prevalencia de enfermedades transmisibles, las relaciones sociales, la calidad de vida de los hogares, entre otros. Cada encuesta tiene una periodicidad determinada y una metodología que busca la representatividad regional o nacional de sus resultados, destacando la Encuesta Demográfica y de Salud Familiar (ENDES) como la de mayor antigüedad (con datos desde 1986) ${ }^{(1)}$.

Los datos (informes y microdatos) de estas encuestas son de acceso libre desde el 2000 y se encuentran disponibles en la página web del INEI (http://iinei.inei.gob.pe/microdatos/). En este sentido, son una alternativa económicamente rentable para que nuevos investigadores desarrollen contenido científico de calidad y que pueda servir como evidencia en la toma de decisiones para la salud pública del país ${ }^{(2-4)}$. Por ello, surge el interés por conocer el uso de las encuestas nacionales peruanas como fuente generadora de investigaciones a lo largo del tiempo.

\footnotetext{
Sociedad Científica de San Fernando, Universidad Nacional Mayor de San Marcos. Lima, Perú.

a Estudiante de medicina

Recibido:06/11/2017 Aprobado:30/11/2017 En línea:05/04/2018
}

Citar como: Ruiz-Maza JC, Pezo-Pezo AM, Soto-Azpilcueta RA. Producción científica en base a cinco encuestas nacionales de Perú. Rev Peru Med Exp Salud Publica. 2018;35(1): 166-7.doi: 10.17843/rpmesp.2018.351.3554.
Se realizó un estudio bibliométrico sobre la producción científica, durante el periodo 1986-2017, en base a la utilización de datos de cinco encuestas desarrolladas en Perú: Encuesta Demográfica y de Salud Familiar (ENDES), Encuesta Nacional de Hogares (ENAHO), Encuesta Nacional de Relaciones Sociales (ENARES), Encuesta Nacional Especializada de Discapacitados (ENEDIS) y Encuesta Nacional de Satisfacción de Usuarios en Salud (ENSUSALUD).

La búsqueda se realizó en Scopus, Medline y SciELO, en la estrategia de búsqueda se utilizó las siguientes palabras clave: "encuesta nacional", "National survey", "DHS", "Encuesta Demográfica y de Salud Familiar","Demographich and health survey", "Demographic and Family Health Survey", "ENDES", "Encuesta Nacional de Hogares" ,"National Household Survey","ENAHO”, "Encuesta Nacional de Relaciones Sociales","national survey on social relations","ENARES", "Encuesta Nacional de Satisfacción de Usuarios en Salud", "National Survey of Satisfaction of Users in Health" "ENSUSALUD", "Encuesta Nacional Especializada de Discapacitados", "Disability National Survey ","ENEDIS”.

Se incluyeron los siguientes tipos de estudios: artículos originales, originales breves, revisiones y cartas al editor. La búsqueda se realizó del 1 al 7 de setiembre de 2017 , fueron categorizados por año de publicación y revista. Los datos fueron tabulados y analizados en Excel 2007.

Ingresaron al estudio 73 publicaciones de los cuales el $93,1 \%$ fueron artículos originales, el $84,9 \%$ fueron publicadas desde el 2000 y el 74,0\% utilizaron los datos de la ENDES. Al revisar la indización de las revistas donde se realizó la publicación se encontró que el 43,8\% estaban en Scopus y Medline. Además, la mayoría de publicaciones se realizó en la Revista Peruana de Medicina Experimental y Salud Pública (17,8\%) (Tabla 1).

Nuestros resultados demuestran el uso de los datos de las encuestas nacionales, principalmente ENDES o ENAHO, en el desarrollo de publicaciones en revistas nacionales e internacionales indizadas en bases de datos con elevados criterios de rigurosidad y calidad ${ }^{(4,5)}$.

La disponibilidad virtual de los datos de encuestas nacionales como la ENDES influye en el nivel de producción científica a través del tiempo ${ }^{(3)}$. Esto también se observa en nuestro estudio y explica la diferencia del nivel de productividad científica de encuestas con mayor antigüedad como ENDES y ENAHO (disponibles desde el 2000) en comparación a las encuestas más recientes como ENARES, ENSUSALUD y ENEDIS (disponibles desde el 2013).

Asimismo, el $40,1 \%$ de estas investigaciones fueron publicadas en solo cinco revistas, dato similar al reportado en estudios previos ${ }^{(3)}$. La elección de una revista esta 